\title{
Pseudo-random graphs and bit probe schemes with one-sided error *
}

\author{
Andrei Romashchenko \\ CNRS (France) \& IITP of RAS (Russia, Moscow)
}

November 10, 2018

\begin{abstract}
We study probabilistic bit-probe schemes for the membership problem. Given a set $A$ of at most $n$ elements from the universe of size $m$ we organize such a structure that queries of type " $x \in A$ ?" can be answered very quickly.

H. Buhrman, P.B. Miltersen, J. Radhakrishnan, and S. Venkatesh proposed a bit-probe scheme based on expanders. Their scheme needs space of $O(n \log m)$ bits. The scheme has a randomized algorithm processing queries; it needs to read only one randomly chosen bit from the memory to answer a query. For every $x$ the answer is correct with high probability (with two-sided errors).

In this paper we show that for the same problem there exists a bitprobe scheme with one-sided error that needs space of $O\left(n \log ^{2} m+\right.$ poly $(\log m))$ bits. The difference with the model of Buhrman, Miltersen, Radhakrishnan, and Venkatesh is that we consider a bit-probe scheme with an auxiliary word. This means that in our scheme the memory is split into two parts of different size: the main storage of $O\left(n \log ^{2} m\right)$ bits and a short word of $\log ^{O(1)} m$ bits that is pre-computed once for the stored set $A$ and "cached". To answer a query " $x \in A$ ?" we allow to read the whole cached word and only one bit from the main storage. For some reasonable values of parameters (e.g., for poly $(\log m) \ll n \ll m$ ) our space bound is better than what can be achieved by any scheme without cached data (the lower bound $\Omega\left(\frac{n^{2} \log m}{\log n}\right)$ was proven in 18]). We obtain a slightly weaker result (space of size $n^{1+\delta}$ poly $(\log m)$ bits and two bit probes for every query) for a scheme that is effectively encodable.

Our construction is based on the idea of naive derandomization, which is of independent interest. First we prove that a random combinatorial object (a graph) has the required properties, and then show that such a graph can be obtained as an outcome of a pseudo-random bits generator. Thus, a suitable graph can be specified by a short seed of a PRG, and we can put an appropriate value of the seed into the cache memory of the scheme.
\end{abstract}

*Supported in part by grants ANR EMC ANR-09-BLAN-0164-01 and NAFIT ANR-08EMER-008-01. 


\section{Introduction.}

We investigate the static version of the membership problem. The aim is to represent a set $A \subset\{1, \ldots, m\}$ by some data structure so that queries " $x \in$ $A$ ?" can be easily replied. We are interested in the case when the number of elements in the set $n=|A|$ is much less than size $m$ of the universe (e.g., $n=\exp \{\operatorname{poly}(\log \log m)\}$ or $\left.n=m^{0.01}\right)$.

In practice, many different data structures are used to represent sets: simple arrays, different variants of height-balanced trees, hash tables, etc. The simplest solution is just an array of $m$ bits; to answer a query " $x \in A$ ?" we need to read a single bit from the memory (the $x$-th bit in the data storage is equal to 1 if $x \in A$ ). However, the size of this data structure is excessive: it requires $m$ bits of memory, while there exist only $\left(\begin{array}{c}m \\ n\end{array}\right)=2^{\Theta(n \log m)}$ different subsets $A$ of size $n$ in the $m$-elements universe.

Nowadays the standard practical solution for the membership problem is a more complex data structure proposed by Fredman, Komlós, and Szemerédi [6]. This scheme is based on perfect hashing; a set is represented as a table of $O(n)$ words (hash-values) of size $\log m$ bits, and a query " $x \in A$ ?" requires to read $O(1)$ words from the memory. The space complexity of this construction is quite close to the trivial lower bound $\Omega\left(\log \left(\begin{array}{l}m \\ n\end{array}\right)\right)$. The asymptotic of the space complexity of this scheme was further improved in [11, 15, 16]. Similar space complexity was achieved in dynamic data structures, which support fast update of the set stored in the database (see, e.g., analysis of the cuckoo hashing scheme in [26, 25). A subtle analysis of the space and bit-probe complexity for the membership problems was given also in 20. (in particular, 20] suggested a membership scheme based on bounded concentrator graphs). Note that all these schemes require to read from the memory $O(\log m)$ bits to answer each query.

Another popular practical solution is Bloom's filter [1]. This data structure requires only $O(n)$ bits, whatever is the size of the universe; to answer a query we need to read $O(1)$ bits from the memory. The drawback of this method is that we can get false answers to some queries. Only false positives answers are possible (for some $x \notin A$ Bloom's filter answers "yes"), but false negatives are not. When this technique is used in practice, it is believed that for a "typical" set $A$ the fraction of false answers should be small. However, in many applications we cannot fix a priori any reasonable probability distribution on the family of all sets $A$ and on the space of possible queries.

An interesting alternative approach was suggested by Harry Buhrman, Peter Bro Miltersen, Jaikumar Radhakrishnan, and Venkatesh Srinivasan [18]. They introduced randomness into the query processing algorithm. That is, the data structure remains static (it is deterministically defined for each set $A$ ), but when a query is processed, we a toss coins and read randomly chosen bit from the memory. In this model, we allow to return a wrong answer with some small probability. Notice the sharp difference with the Bloom's filter: Now we must correctly reply to the query " $x \in A$ ?" with probability close to 1 for each $x$.

Buhrman, Miltersen, Radhakrishnan, and Venkatesh investigated both two- 
sided and one-sided errors. In this paper we will concentrate mostly on one-sided errors: if $x \in A$, then the answer must be always correct, and if $x \notin A$, then a small probability of error is allowed.

Recall that a trivial information-theoretic bound shows that the size of the structure representing a set $A$ cannot be less that $\log \left(\begin{array}{c}m \\ n\end{array}\right)=\Omega(n \log m)$ bits. Surprisingly, this bound can be achieved if we allow two-sided errors and use only single bit probe for each query. This result was proven in [18. We refer to the scheme proposed their as the BMRV-scheme:

Theorem 1 (two-sided BMRV-scheme, [18]) For any $\varepsilon>0$ there is a scheme for storing subsets $A$ of size at most $n$ of a universe of size $m$ using $O\left(\frac{n}{\varepsilon^{2}} \log m\right)$ bits so that any membership query "Is $x \in A$ ?" can be answered with error probability less than $\varepsilon$ by a randomized algorithm which probes the memory at just one location determined by its coin tosses and the query element $x$.

The size of the memory achieved in this theorem is only a constant factor greater than the best possible. In fact, the trivial lower bound $\log \left(\begin{array}{c}m \\ n\end{array}\right)$ can be improved: the less is the probability of an error of the scheme, the more memory we need.

Theorem 2 (lower bound, [18]) (a) For any $\varepsilon>0$ and $\frac{n}{\varepsilon}<m^{1 / 3}$, any $\varepsilon$ error randomized scheme which answers queries using one bitprobe must use space $\Omega\left(\frac{n}{\varepsilon \log 1 / \varepsilon} \log m\right)$.

(b) Any scheme with one-sided error $\varepsilon$ that answers queries using at most one bitprobe must use $\Omega\left(\frac{n^{2}}{\varepsilon^{2} \log (n / \varepsilon)} \log m\right)$ bits of storage.

Note that for one-sided error schemes the known lower bound is much stronger. Part (b) of the theorem above implies that we cannot achieve the size of space $O(n \log m)$ with a one probe scheme and one-sided error. However we can get very close if we allow $O(1)$ probes instead of a single probe:

Theorem 3 (one-sided BMRV-scheme, [18]) Fix any $\delta>0$. There exists a constant $t$ such that the following holds: There is a one-sided $\frac{1}{3}$-error randomized scheme that uses space $O\left(n^{1+\delta} \log m\right)$ and answers membership queries with at most $t$ probes.

The constructions in [18 is not explicit: given the list of elements $A$, the corresponding scheme is constructed (with some brute force search) in time $2^{\text {poly }(m)}$. Moreover, each membership query requires exponential in $m$ computations.

The crucial element of the constructions in Theorem 1 is an unbalanced expander graph. Existence of a graph with required parameters was proven in [18] probabilistically. We know that such a graph exists and we can find it by brute force search, but we do not know how to construct it explicitly. In case we have an effective construction of an expander with good parameters, we will get a practical variant of the BMRV-scheme. This scheme could be also generalized to build more complex data structures (see [23] for a construction 
of a dictionary data structure based on the BMRV-scheme and some explicit expanders).

Since Bassalygo and Pinsker defined expanders [2, 3, many explicit (and poly-time computable) constructions of expander graphs were discovered, see a survey 27. However, most of the known constructions are based on the spectral technique that is not suitable to get an expander of degree $d$ with an expansion parameter greater than $d / 2$, see [13]. This is not enough for the construction used in the proof of Theorem 1 in [18; we need there a graph with expansion parameter close to $d$.

There are only very few effective constructions of unbalanced graph with large expansion parameter. One of the known constructions was suggested by Capalbo et al in 21; its parameters are close to the optimal values if the size of the right part of the graph is constant times less than the size of the left part of the graph. However, in the BMRV-scheme we need a very unbalanced expander, i.e., a graph where the right part of the graph is much less than the left part; so, the technique from 21] seems to be not suitable here. Some explicit version of the BMRV-scheme was suggested in 24] (this construction involves Trevisan's extractor; note that Trevisan's extractor is known to be a good highly unbalanced expander, [19]). The best known explicit construction of a highly unbalanced expander graph was presented in 31. It is based on the Parvaresh-Vardy code with an efficient list decoding. Thanks to the special structure if this expander, it enjoys some nice property of effective decoding. Using this technique, the following variant of Theorem 1 can be proven:

Theorem 4 ([31]) For any $\delta>0$ there exists a scheme for storing subset $A$ of size at most $n$ of a universe of size $m$ using $n^{1+\delta} \cdot \operatorname{poly}(\log m)$ bits so that any membership query can be answered with error probability less than $\varepsilon$ by a randomized algorithm which probes the memory at one location determined by its coin tosses and the query element $x$.

Given the list of elements A, the corresponding storing scheme can be constructed in time poly $(\log m, n)$. When the storing scheme is constructed, a query for an element $x$ can be calculated in time poly $(\log m)$.

In Theorems 1, 3. 4, a set $A$ is encoded into a bit string, and when we want to know if $x \in A$, we just read from this string one randomly chosen bit (or $O(1)$ bits in Theorem (3). The obtained information is enough to decide whether $x$ is an element of the set. Let us notice that in all these computations we implicitly use more information than just a single bit extracted from the memory. To make a query to the scheme and to process the retrieved bit, we need to know the parameters of the scheme: the size $n$ of the set $A$, the size $m$ of the universe, and the allowed error probability $\varepsilon$. This auxiliary information is very short (it takes only $\log (m / \varepsilon)$ bits), and it does not depend on the stored set $A$. We assume that this information is somehow hardwired into the bitprobe scheme (we shall say that this information is cached in advance by the algorithms that processes queries).

In this paper we consider a more liberal model, where some small information 
cached by the scheme can depend not only on $n, m$, and $\varepsilon$, but also on the set $A$. Technically, the data stored in our scheme consists of two parts of different size: a small cached string $C$ of length poly $(\log m)$, and a long bit string $B$ of length $n \cdot \operatorname{poly} \log (m)$. Both these strings are prepared for a given set $A$ of $n$ elements (in the universe of size $m$ ). When we need to answer a query " $x \in A$ ?", we use $C$ to compute probabilistically a position in $B$ and read there one bit. This is enough to answer whether $x$ is an element of $A$, with a small one-sided error:

Theorem 5 Fix any constant $\varepsilon>0$. There exists a one-sided $\varepsilon$-error randomized scheme that includes a string $B$ of length $O\left(n \log ^{2} m\right)$ and an auxiliary word $C$ of length poly $(\log m)$. We can answer membership queries " $x \in A$ ?" with one bit probe to $B$. For $x \in A$ the answer is always correct; for each $x \notin A$ probability of error is less than $\varepsilon$.

The position of the bit probed in $A$ is computed from $x$ and the auxiliary word $C$ in time poly $(\log m)$.

Remark 1: Schemes with 'cached' auxiliary information that depends on $A$ (not only on its size $n=|A|$ and the size $m$ of the universe) make sense only if the cached information is very small. Indeed, if the size of the cached data is about $\log \left(\begin{array}{c}m \\ n\end{array}\right)$ bits, then we can put there the list of all elements of $A$, so the problem becomes trivial. Since in our construction we need cached information of size poly $(\log m)$ bits, the result is interesting when poly $(\log m) \ll n \ll m$, e.g., for $n=\exp \{\operatorname{poly}(\log \log n)\}$. Note that by Theorem 2 the space size $O\left(n \log ^{2} m\right)$ with one-sided error cannot be achieved by any schemes without cached auxiliary information that depends on $A$.

Remark 2: The model of data structures with cached memory looks useful for practical applications. Indeed, most computer systems contain some hierarchy of memory levels: CPU registers and several levels of processor caches, then random access memory, flash memory, magnetic disks, remote network-accessible drives, etc. Each next level of memory is cheaper but slower. So, it is interesting to investigate the tradeoff between expensive and fast local memory and cheap and slow external memory. There is a rich literature on algorithms with external memory, see, e.g, surveys [17, 28. Thus, the idea of splitting the data structure into 'cached' and 'remote' parts is very natural and quite common in computer science. However, tradeoff between local and external memory is typically studied for dynamic data structures. The same time, it is not obvious that fast cache memory of negligible size can help to process queries in a static data structure. Since a small cache 'knows' virtually nothing about most objects in the database, at first sight it seems to be useless. However, Theorem 5 shows that even a very small cache can be surprisingly efficient.

Remark 3: In the proof of Theorem 5 we derandomize a probabilistic proof of existence of some kind of expander graphs. In many papers derandomization of probabilistic arguments involves highly sophisticated $a d-h o c$ techniques. But we do derandomization in rather naive and straightforward way: take a value of a suitable pseudo-random bits generator and check that with high probability 
(i.e., for most values of the seed) a pseudo-random objects enjoys the required property. In fact, we observe that several types of generators fit our construction. Since the required property of a graph can be tested in $\mathrm{AC}^{0}$, we can use the classic Nisan-Wigderson generator or (thanks to the recent result of Braverman [29]) any polylog-independent function. Also the required property of a pseudo-random graph can be tested by a machine with logarithmic space. Hence, we can use Nisan's generator [10]. Our idea of employing pseudo-random structures is quite similar to the construction of pseudo-random hash-functions in [30. We stress that we do not need any unproven assumptions to construct all these generators.

In Theorem 5 we construct a scheme such that decoding is effective: when the scheme is prepared, we can answer queries " $x \in A$ ?" in time polynomial in $\log m$. However the encoding (preparing the database and the auxiliary word for a given set $A$ ) runs in expected time poly $(m)$. We assume that $n \ll m$, and the time polynomial in $m$ seems to be too long. It is natural to require that encoding of the scheme runs in time $\operatorname{poly}(n, \log m)$ (i.e., polynomial in the size of the encoded set $A$ and the size of an index of each element in the universe). The next theorem claims that the encoding time can be reduced if we slightly increase the space of the scheme:

Theorem 6 The scheme from theorem 5 can be made effectively encodable in the following sense. Fix any constants $\varepsilon, \delta>0$. There exists randomized scheme that includes a bit string $B$ of length $n^{1+\delta}$ poly $(\log m)$ and an auxiliary word $C$ of length $\operatorname{poly}(\log m)$. We can answer membership queries " $x \in A$ ?" with two bits probe to $B$. For $x \in A$ the answer is always correct; for $x \notin A$ probability of error is less than $\varepsilon$.

The position of the bit probed in $A$ is computed by $x$ and the auxiliary word $C$ in time poly $\log (m)$. Given A, the entire scheme (the string $B$ and the word $C)$ can be computed probabilistically in average time $\operatorname{poly}(n, \log m)$.

The rest of the paper is organized as follows. In Section 2 we remind the main ideas in the BMRV-scheme. We prove Theorem 5 in Section 3, and Theorem 6 in Section 4. In Section 5 we show that the proof of Theorem 2 (a) mutatis mutandis can be applied to our model with small cached memory. In Conclusion we discuss some open questions.

\section{How BMRV-scheme works.}

Let us explain the main ideas of the proof of Theorem 1 in [18. The construction is based on highly unbalanced expanders.

Definition 1 A bipartite graph $\mathcal{G}=(L, R, E)$ (with left part $L$, right part $R$ and set of edges $E)$ is called $(m, s, d, k, \delta)$-expander if $L$ consists of $m$ vertices, $R$ consists of $s$ vertices, degree of each vertex in $L$ is equal to $d$, and for each subset of vertices $A \subset L$ of size at most $k$ the number of neighbors is at least $(1-\delta) d|A|$. 
We use a standard notation: for a vertex $v$ we denote by $\Gamma(v)$ the set of its neighbors; for a set of vertices $A$ we denote by $\Gamma(A)$ the set of neighbors of $A$, i.e., $\Gamma(A)=\cup_{v \in A} \Gamma(v)$. So, the definition of expanders claims that for all small enough sets $A$ of vertices in the left part of the graph, $|\Gamma(A)| \geq(1-\delta) d|A|$ (the maximal size of $|\Gamma(A)|$ is obviously $d|A|$, since degrees of all vertices on the left are equal to $d$ ). The argument below is based on the following combinatorial property of an expander:

Lemma 1 (see [21]) Let $\varepsilon$ be a positive number, and $\mathcal{G}$ be an $(m, s, d, k, \delta)$ expander with $\delta \leq \varepsilon / 4$. Then for every subset $A \subset L$ such that $|A| \leq k / 2$, the number of vertices $x \in L \backslash A$ such that

$$
|\Gamma(x) \cap \Gamma(A)| \geq \varepsilon d
$$

is not greater than $|A| / 2$.

Let $\mathcal{G}$ be a $(m, s, d, k, \delta)$-expander with $\delta<\varepsilon / 4$. The storage scheme is defined as follows. We identify a set $A \subset\{1, \ldots, m\}$ of size $n(n \leq k / 2)$ with a subset of vertices in the left part of the graph. We will represent it by some labeling (by ones and zeros) on the vertices of the right part of the graph. We do it in such a way that the vast majority (at least $(1-\varepsilon) d$ ) of neighbors of each vertex $v$ from the left part of the graph correctly indicate whether $v \in A$. More precisely, if $v \in A$ then at least $(1-\varepsilon) d$ of its neighbors in $R$ are labeled by 1 ; if $v \in L \backslash A$ then at least $(1-\varepsilon) d$ of its neighbors in $R$ are labeled by 0 . Thus, querying a random neighbor of $v$ will return the right answer with probability $>1-\varepsilon$.

It remains to explain why such a labeling exists. In fact, it can be constructed by a simple greedy algorithm. First, we label all neighbors of $A$ by 1 , and the other vertices on the left by 0 . This labeling classifies correctly all vertices in $A$. But it can misclassify some vertices outside $A$ : some vertices in $L \backslash A$ can have too many (more than $\varepsilon d$ ) neighbors labeled by 1 . Denote by $B$ the set of all these "erroneous" vertices. We relabel all their neighbors, i.e., all vertices in $\Gamma(B)$ to 0 . This fixes the problem with vertices outside $A$, but it can create problems with some vertices in $A$. We take the set of all vertices in $A$ that became erroneous (i.e., vertices in $A$ that have at least $\varepsilon d$ neighbors in $\Gamma(B)$ ), and denote this set of vertices by $A^{\prime}$. Then, we relabel all $\Gamma\left(A^{\prime}\right)$ to 1 . This operation create new problems in some set of vertices $B^{\prime} \subset B$, we relabel $\Gamma\left(B^{\prime}\right)$ to 0 , etc. In this iterative procedure we get a sequence of sets

$$
A \supset A^{\prime} \supset A^{\prime \prime} \supset \ldots
$$

whose neighbors are relabeled to 1 on steps $1,3,5, \ldots$ of the algorithm, and

$$
B \supset B^{\prime} \supset B^{\prime \prime} \supset \ldots
$$

whose neighbors are relabeled to 0 on iterations $2,4,6, \ldots$ respectively. Lemma 1 guarantees that the number of the erroneous vertices on each iteration reduces by a factor of $2\left(|B| \leq|A| / 2,\left|A^{\prime}\right| \leq|B| / 2\right.$, etc.). Hence, in $\log m$ steps the procedure terminates. 
To organize a storing scheme (and to estimate its size) for a set $A$ of size $n$ in the universe of size $m$, we should construct an $(m, s, d, k=2 n, \delta=\varepsilon / 4)$ expander. Parameters $m, k, \delta$ of the graph are determined directly by the parameters of the desired scheme (by the size of $A$ and the universe and the allowed error probability $\varepsilon$ ). We want to minimize the size of the left part of the graph $s$, which is the size of the stored data. Existence of expanders with good parameters can be proven by probabilistic arguments:

Lemma $2([\mathbf{1 8})$ For all integers $m, n$ and real $\varepsilon>0$ there exists an $(m, s=$ $\left.O\left(\frac{n \log m}{\varepsilon^{2}}\right), d=\frac{\log m}{\varepsilon}, n, \varepsilon\right)$-expander. Moreover, the vast majority of bipartite graphs with $n$ vertices on the left, $s=\frac{100 n \log m}{\varepsilon^{2}}$ vertices on the right, and degree $d=\frac{\log m}{\varepsilon}$ at all vertices on the left are such expanders.

Given the parameters $m, n, \varepsilon$, we can find an $\left(m, O\left(\frac{n \log m}{\varepsilon^{2}}\right), \frac{\log m}{\varepsilon}, n, \varepsilon\right)$-expander by brute force search. This can be done by a deterministic algorithm in time $2^{\text {poly }(m / \varepsilon)}$. Hence, we can construct the bit-probe structure defined above in exponential time. Moreover, when the structure is constructed and we want to answer a query " $x \in A$ ?", we need to read only one bit from the stored bit string. But to select the position of this bit we need again to reconstruct the expander graph, which requires exponential computations. We could keep the structure of the computed graph in "cache" (compute the graph once, and then re-use it every time a new query should be answered). However, in this case the size of the "cached data" (the size of the graph) becomes much greater than $m$, which makes the bit-probe scheme meaningless (it is cheaper to store $A$ as a trivial $m$-bits array).

In 31] a nice and very powerful explicit construction of expanders was suggested:

Theorem 7 ([31]) Fix an $\varepsilon>0$ and $\delta>0$. For all integers $m, n$ there exists an explicit $\left(m, s=n^{1+\delta} \cdot \operatorname{poly}(\log m), d=\operatorname{poly}(\log m), n, \varepsilon\right)$-expander such that for an index of a vertex $v$ from the left part (a binary representation of an integer between 1 and $m$ ) and an index of an outgoing edge (a binary representation of an integer between 1 and $d$ ), the corresponding neighbor on the right part of the graph (an integer between 1 and s) can be computed in time polynomial in $\log m$.

Also, the following effective decoding algorithm exists. Given a set of vertices $T$ from the right part of the graph, we can compute the list of vertices in the left part of the graph that have at least (4عd) neighbors in $T$, i.e.,

$$
S=\{v:|\Gamma(v) \cap T| \geq 4 \varepsilon d\},
$$

in time $\operatorname{poly}(|S|, n, \log m)$.

Theorem 4 is proven by plugging the expander from Theorem 7 in the general scheme explained above, see details in [31. 


\section{Proof of Theorem 5 .}

\subsection{Refinement of the property of $\varepsilon$-reduction.}

The construction of a bit-probe scheme for a set $A$ of size $n$ in the $m$-elements universe (with probability of an error bounded by some $\varepsilon$ ) explained in the previous section involves an $(m, s, d, k, \delta)$-expander with $s=O\left(\frac{n}{\varepsilon^{2}} \log m\right)$ and $d=O\left(\frac{1}{\varepsilon} \log m\right)$. Such a graph contains $d m$ edges (degree of each vertex on the left is $d)$. The list of all its edges can be specified by a string of $d m \log s$ bits: we sort all edges by their left ends, and specify for each edge its right end. Denote the size of the description of this graph by $N=d m \log s$.

In what follows we will assume that number $s$ is a power of 2 (this will increase the parameters of the graph only by a factor at most 2). So, we may assume that every string of $N(m, s, d)=d m \log s$ bits specifies a bipartite graph with $m$ vertices on the left, $s$ vertices on the right and degree $d$ on the left. Lemma 2 claims that most of these bits string of length $N$ describe an $(m, s, d, k, \delta)$-expander. By Lemma 1, if a graph is an expander with these parameters, then for $\varepsilon=4 \delta$ and for every set $A \subset L$ of size less than $k / 2$ the following reduction property holds:

Combinatorial Property 1 (E-reduction property) For every subset $A \subset$ $L$ such that $|A| \leq k / 2$, the number of vertices $x \in L \backslash A$ such that

$$
|\Gamma(x) \cap \Gamma(A)| \geq \varepsilon d
$$

is not greater than $|A| / 2$.

This property was the main ingredient of the BMRV-scheme. In our bit-probe scheme we will need another variant of Property 1 .

Combinatorial Property 2 (strong $\varepsilon$-reduction) Let $\mathcal{G}=(L, R, E)$ be a bipartite graph, and $A \subset L$ be a subset of vertices from the left part. We say that the strong $\varepsilon$-reduction property holds for $A$ in this graph if for all $x \in L \backslash A$

$$
|\Gamma(x) \cap \Gamma(A)| \leq \varepsilon d .
$$

Lemma 3 Fix an $\varepsilon>0$. For all integers $m, n$, for every $A \subset\{1, \ldots, m\}$ of size $n$ there exists a bi-partite graph $\mathcal{G}=(L, R, E)$ such that

- $|L|=m$ (the size of the left part);

- $|R|=2 d^{2} n=O\left(n \log ^{2} m\right)$ (the size of the right part);

- degree of each vertex in the left part is $d=\frac{2 \log m}{\varepsilon}=O(\log m)$;

- the property of strong $\varepsilon$-reduction holds for the set A (we identify it with a subset of vertices in left part of the graph).

Moreover, the property of strong E-reduction for A holds for the majority of graphs with the parameters specified above. 
The order of quantifiers is important here: we do not claim that in a random graph the strong $\varepsilon$-reduction property holds for all $A$; we say only that for every $A$ the strong $\varepsilon$-reduction is true in a random graph.

Proof of lemma: Let $v$ be any vertex in $L \backslash A$. We estimate probability that at least $\varepsilon d$ neighbors of $x$ are at the same time neighbors of $A$ (assuming that all edges are chosen at random independently). There are $\left(\begin{array}{c}d \\ \varepsilon d\end{array}\right)$ choices of $\varepsilon d$ vertices among all neighbors of $v$. Hence,

$\operatorname{Prob}[|\Gamma(v) \cap \Gamma(A)| \geq \varepsilon d] \leq\left(\begin{array}{c}d \\ \varepsilon d\end{array}\right) \cdot\left(\frac{|\Gamma(A)|}{|R|}\right)^{\varepsilon d} \leq d^{\varepsilon d} \cdot\left(\frac{d n}{2 d^{2} n}\right)^{\varepsilon d}=\left(\frac{1}{2}\right)^{2 \log m}$

This probability is less than $1 / m^{2}$ (for each vertex $v$ ). So, the expected number of vertices $v \in L$ such that $|\Gamma(v) \cap \Gamma(A)| \geq \varepsilon d$, is less than $1 / m<1 / 2$. Hence, the strong $\varepsilon$-reduction property holds for $A$ for more than $50 \%$ of graphs.

\subsection{Testing the property of strong $\varepsilon$-reduction.}

Lemma 3 implies that a graph with the strong reduction property for $A$ exists. Given $A$, we can find such a graph by brute force search. But we cannot use such a graph in our bit-probe scheme even if we do not care about computation complexity: the choice of the graph depends on $A$, and the size of the graph is too large to embed it into the scheme explicitly. We need to find a suitable graph with a short description. We will do it using pseudo-random bits generators ('pseudo-random' graphs will be parameterized by the seed of a generator).

Property 2 is a property of a graph and of a set of vertices $A$ in this graph. We can interpreted it as a property of an $N$-bits string (that determines a graph) and some $A \subset\{1, \ldots, m\}$. Lemma 3 claims that for every $A$, for a randomly chosen graph (a randomly chosen $N$-bits string) with high probability the strong reduction property is true. We want to show that the same is true for a pseudorandom graph. At first, we observe that the strong reduction property can be tested by an $\mathrm{AC}^{0}$ circuit (a Boolean circuit of bounded depth, with polynomial number of gates and, or with unbounded fan-in, and negations).

Indeed, we need to check for each vertex $v \in L \backslash A$ that the number of vertices in $\Gamma(v) \cap \Gamma(A)$ is not large. For each vertex $w$ in the right part of the graph we can compute by an $\mathrm{AC}^{0}$-circuit whether $w \in \Gamma(A)$ :

$$
\bigvee_{u \in A} \bigvee_{i \leq d}[\text { the } i \text {-th neighbor of } u \text { is } w]
$$

(the condition in the square brackets is a statement about one edge in a graph, i.e., it is a conjunction of $O(\log N)$ bits and negations of bits from the representation of this graph). So, for each $v \in L \backslash A$ and for $i=1, \ldots, d$ we can compute whether the $i$-th neighbor of $v$ belongs to $\Gamma(A)$ :

$$
b_{v, i}=\bigvee_{w \in R}([\text { the } i \text {-th neighbor of } v \text { is } w] \&[w \in \Gamma(A)])
$$


It remains to 'count' for each $v \in L \backslash A$ the number of neighbors in $\Gamma(A)$ and compute the thresholds

$$
\operatorname{Th}\left(b_{v, 1}, \ldots, b_{v, d}\right)= \begin{cases}1, & \text { if } b_{v, 1}+\cdots+b_{v, d} \geq \varepsilon d, \\ 0, & \text { otherwise. }\end{cases}
$$

In $\mathrm{AC}^{0}$ we cannot compute thresholds with linear number of inputs (e.g., the majority function is not in $\mathrm{AC}^{0}$, see [8]). However, we need threshold functions with only logarithmic number of inputs. Such a function can be represented by a CNF of size $2^{O(d)}=\operatorname{poly}(N)$.

Then, we combine together these thresholds for all $v \in L \backslash A$, and get an $\mathrm{AC}^{0}$-circuit that tests the property of strong $\varepsilon$-reduction.

\subsection{Pseudo-random graphs.}

We need to generate a pseudo-random string of $N$ bits that satisfies the strong $\varepsilon$-reduction property (for some fixed set $A$ ). We know that (i) by Lemma 3 , for a uniformly distributed random string this property is true with high probability, and (ii) this property can be checked in $\mathrm{AC}^{0}$. It remains to choose a pseudorandom bits generator that fools this particular $\mathrm{AC}^{0}$-circuit. There exist several generators that fools such distinguishers. Below we mention three different solutions.

Remark: We can test by an $\mathrm{AC}^{0}$-circuit Property 2 for every fixed set $A$ but not for all sets $A$ together.

The first solution: the generator of Nisan and Wigderson. The classic way to fool an $\mathrm{AC}^{0}$ circuit is the Nisan-Wigderson generator:

Theorem 8 (Nisan-Wigderson generator, [12]) For every constant c there exists an explicit family of functions

$$
G_{m}:\{0,1\}^{\text {poly }(\log N)} \rightarrow\{0,1\}^{N}
$$

such that for for any family of circuits $C_{N}$ (with $N$ inputs) of polynomial in $N$ size and depth $c$, the difference

$$
\left|\operatorname{Prob}_{y}\left[C_{N}(y)=1\right]-\operatorname{Prob}_{z}\left[C_{N}\left(G_{m}(z)\right)=1\right]\right|
$$

tends to zero (faster than $1 / \operatorname{poly}(N)$ ).

The generator is effective: generator's value $G_{m}(x)$ can be computed from a given $x$ in time poly $(\log N)$.

From this theorem and Lemma 3 it follows that for each $A \subset\{1, \ldots, m\}$ of size at most $n$, for most values of the seed of the Nisan-Wigderson generator $G_{m}$, a pseudo-random graph $G_{m}(x)$ satisfies the strong $\varepsilon$-expansion property for $A$.

The second solution: polylog-independent strings. M. Braverman proves that all polylog-independent functions fool $\mathrm{AC}^{0}$-circuits: 
Theorem $9([29])$ Let $\mathcal{C}$ be a Boolean circuit of depth $r$ and size $M, \varepsilon$ be a positive number, and

$$
D=\left(\log \frac{M}{\varepsilon}\right)^{\kappa r^{2}}
$$

(for some absolute constant $\kappa$ ). Then $\mathcal{C}$ cannot distinguish between the uniform distribution $U$ and any $D$-independent distribution $\mu$ on its inputs:

$\mid \operatorname{Prob}_{\mu}[\mathcal{C}(x)$ accepts a $\mu$-random $x]-\operatorname{Prob}_{U}[\mathcal{C}(x)$ accepts a $U$-random $x] \mid<\varepsilon$.

It follows that instead of the Nisan-Wigderson generator we can take any $\left(\log ^{c} n\right.$ )-independent function (for large enough constant $c$ ). The standard way to generate $r$-independent bits (in our case we need $r=\log ^{c} n$ ) is a polynomial of degree $r$ over a finite field of size about $N$. Seeds of this 'pseudo-random bits generator' are coefficients of a polynomial. Also, other (more computationally effective) constructions of polylog-independent functions can be used. E.g., the construction from [9, 14, provides a family of $\left(\log ^{c} n\right)$-independent functions with very fast evaluation algorithm, and each function is specified by poly $(\log n)$ bits (so, the size of the seed is again poly-logarithmic).

The third solution: the generator of Nisan. The property of strong $\varepsilon$-reduction can be tested by a Turing machine with logarithmic working space. Technically we need a machine with

- advice tape: read-only, two-way tape, where the list of elements of $A$ is written;

- input tape: read-only tape with random (or pseudo-random) bits, with logarithmic number of passes (the machine is allowed to pass along the input on this tape only $O(\log N)$ times);

- index tape: read-only, two-way tape with logarithmic additional information;

- work tape that is two-way and read-write; the zone of the working tape is restricted to $O(\log N)$.

We interpret the content of the input tape as a list of edges of a random (or pseudo-random) graph $\mathcal{G}=(L, R)$. The content of the index tape is understood as an index of a vertex $v \in L \backslash A$. The machine reads the bits from the 'input tape' (understood as a list of edges of a random graph) and checks that the vast majority of neighbors of $v$ does not belong to the set of neighbors of $A$. The machine needs to read the input tape $2 d=O(\log N)$ times (where $d$ is degree of $v$ ): on the first pass we find the index of the first neighbor of $v$; on the second pass we check whether this neighbor of $v$ is incident to any vertex of $A$; then we find the second neighbor of $v$, check whether it is is incident to any vertex of $A$, etc. The machine accepts the input if $|\Gamma(v) \cap \Gamma(A)|<\varepsilon d$.

We can use Nisan's generator [10] to fool this machine. Indeed, this checking procedure fits the general framework of [22], where Nisans generator was used 
to derandomize several combinatorial constructions. The only nonconventional feature in our argument is that the input tape is not read-once: we allow to read the tape with random bits logarithmic number of times 1 . But we can apply Nisan's technique for a machine that reads random bits several times. David, Papakonstantinou, and Sidiropoulos observed (see [33]) that a log-space machine with logarithmic (and even poly-logarithmic) number of passes on the input tape is fooled by Nisan's generator with a seed of size poly $(\log N)$.

Now we are ready to prove Theorem 5 . We fix an $\varepsilon>0$ and a set $A \subset$ $\{1, \ldots, m\}$ of size m. Let $G_{m}$ be one of the pseudo-random bits generators discussed above. For all these generators, for most values of the seed $z$ the values $G_{m}(z)$ encodes a graph such that the strong $\varepsilon$-reduction property holds for $A$. Let us fix one of such seeds. We label by 1 all vertices in $\Gamma(A)$ and by 0 all other vertices in $R$ in the graph encoded by the string $G_{m}(z)$.

The seed value $z$ makes the "auxiliary word" $C$, and the specified above labeling of the right part of the graph makes the bit string $B$. To answer a query " $x \in A$ ?" we take a random neighbor of $x$ in the graph and check its label. If the label is 1 , we answer " $x \in A$ "; otherwise " $x \notin A$ ".

If $x \in A$, then there are no errors, since all neighbors of $A$ are labeled by 1. If $x \notin A$, then probability of an error is bounded by $\varepsilon$ because of the strong $\varepsilon$-reduction property. We can answer a query in time poly $(\log m)$ since the generators under consideration are effectively computable.

\subsection{Complexity of encoding}

The disadvantage of this construction is non-effective encoding procedure. We know that for most seeds $z$ the corresponding graph $G_{m}(z)$ enjoys the strong $\varepsilon$-reduction property. However, we need the brute force search over all vertices $v \in L \backslash A$ (polynomial in $m$ but not in $\log m$ ) to check this property for any particular seed. Thus, we have a probabilistic encoding procedure that runs in expected time poly $(m)$ : we choose random seeds until we find one suitable for the given $A$.

In the next section we explain how to make the encoding procedure more effective (in expected time poly $(n, \log m)$ ) for the following price: we will need a slightly greater size of the data storage, and we will take 2 bit probes instead of one at each query.

\section{Proof of theorem 6: effective encoding.}

To obtain a scheme with effective encoding and decoding we combine two constructions: the explicit expander from [31] and a pseudo-random graph from the previous section.

\footnotetext{
${ }^{1}$ The same argument can be presented in a more standard framework, with a read-once input tape and an index tape of poly-logarithmic size. However, we believe that the argument becomes more intuitive when we allow many passes on the input tape.
} 
We fix an $n$-element set $A$ in the universe $\{1, \ldots, m\}$. Now we construct two bipartite graphs $\mathcal{G}_{1}$ and $\mathcal{G}_{2}$ that share the same left part $L=\{1, \ldots, m\}$. The first graph is the explicit $\left(m, s=n^{1+\delta} \cdot \operatorname{poly}(\log m), d=\operatorname{poly}(\log m), n, \varepsilon\right)$ expander $\mathcal{G}_{1}=\left(L, R_{1}, E_{1}\right)$ from 31 with an effective decoding algorithm. We do the first two steps from the encoding procedure of the BMRV-scheme explained in Section 2 At first we label all vertices in $\Gamma(A)$ by 1 and other vertices by 0 . Denote the corresponding labeling (which is a $n^{1+\delta} \cdot \operatorname{poly}(\log m)$-bits string) by $B_{1}$. Then, we find the list of vertices outside $A$ that have too many 1-labeled neighbors:

$$
W:=\{v \in L \backslash A:|\Gamma(x) \cap \Gamma(A)| \geq \varepsilon d\} .
$$

We do not re-label neighbors of $W$, but we will use this set later (to find $W$ effectively, we need the property of effective decoding of the graph).

Let $v \in L$ be a vertex in the left part of the graph. There are three different cases:

- if $v$ belongs to $A$ then all neighbors of $v$ are labeled by 1 ;

- if $v$ does not belong to $A \cup W$, then a random neighbor of $x$ with probability $>(1-\varepsilon) d$ is labeled by 0 ;

- if $v$ belongs to $W$, we cannot say anything certain about labels of its neighbors.

Thus, if we take a random neighbor of $v$ and see label 0 in $B_{1}$, then we can say that this point does not belong to $A$. If we see label 1 , then a more detailed investigation is needed. This investigation will involve the second part of the scheme, which we define below.

Now our goal is to distinguish between $A$ and $W$. To this end, we take a pseudo-random graph $\mathcal{G}_{2}=\left(L, R_{2}, E_{2}\right)$ specified by a value of a pseudo-random bits generator $G_{m}(z)$ (any of the generators discussed in the previous section is suitable). We need a restricted on $W$ version of the strong $\varepsilon$-reduction property:

For every $v \in W$, at most $\varepsilon d$ vertices in $\Gamma(v)$ belong to $\Gamma(A)$.

Set $W$ is of size at most $|A| / 2$ (Lemma1), and it can be effectively computed from $A$ (effective decoding property of the graph $\mathcal{G}_{1}$ ). Hence, for a given $z$ we can check the property above in time $\operatorname{poly}(n, \log m)$. We know that for the majority of seeds $z$, the graph $G_{m}(z)$ satisfies the strong $\varepsilon$-reduction property, i.e., all vertices outside $A$ have at most $\varepsilon d$ neighbors in $\Gamma(A)$. Though we cannot effectively check this general property (we cannot check it effectively for all vertices in the universe), we are able to check its restricted version (i.e., only for vertices in $W$ ).

Thus, in average time $\operatorname{poly}(n, \log m)$ we can probabilistically find some seed $z$ such that the restricted (on $W$ ) version of the strong $\varepsilon$-reduction property is true. In the corresponding graph $\mathcal{G}_{2}$ we label by 1 all vertices in $\Gamma(A)$, and by 0 all vertices of the right part of the graph outside $\Gamma(A)$. We denote this labeling (a $O\left(m \log ^{2} n\right)$-bits string) by $B_{2}$ and take it as the second part of the data storage. The corresponding seed value $z$ is taken as 'cached' memory. 
The decoding procedure works as follows. Given $x \in\{1, \ldots, m\}$, we take its random neighbor in both constructed graphs and look at their labels (bits from $B_{1}$ and $B_{2}$ respectively).

- if the first label is 0 , we say that $x \notin A$;

- if the first label is 1 and the second bit is 0 then we say that $x \notin A$.

- if both labels are is 1 then we say that $x \in A$.

If $x \notin A \cup W$, then by the definition of $W$ we know that the procedure above with probability $>(1-\varepsilon)$ returns the correct answer. If $x \in A$, then by construction, both labels are equal to 1 , and the procedure returns the correct answer with probability 1 . If $x \in W$, then we have no guarantee about labels in $B_{1}$; but from the restricted strong reducibility property it follows that with probability $>(1-\varepsilon)$ the second label is 0 . Thus, we have one-sided error probability bounded by $\varepsilon$.

\section{A lower bound for schemes with cached mem- ory.}

In 18 the lower bound $\Omega\left(n^{2} \log m\right)$ was proven for one-probe schemes with one-sided errors. This result cannot be applied to schemes with small "cached" memory dependent on $A$. In fact, our scheme of size $\Omega(n \log m)$ with a cache of size poly $(\log m)$ bits (from Theorem [5) is below this bound for $n \gg \log ^{O(1)} m$.

On the other hand, the proof of the lower bound $\Omega\left(\frac{n}{\varepsilon \log (1 / \varepsilon)} \log m\right)$ (theorem 2 in [18) with minimal changes works for schemes with cached data if the size of the pre-computed and cached information is much less than $n \log m$ :

Theorem 10 We consider randomized schemes that store sets of $n$ elements from a universe of size $m$, with two-parts memory (the cached memory of size poly $(\log m)$ and the main storage).

For all constant $\varepsilon<1$, if poly $(\log m) \ll n \ll \sqrt[3]{m}$, then any such scheme with error probability $\varepsilon$ (possibly with two-sided errors) that answers queries using cached memory of size poly $\log (m)$ and one bit probe to the main storage, must use space $\Omega\left(\frac{n}{\varepsilon \log (1 / \varepsilon)} \log m\right)$.

Proof: We follow the arguments from theorem 2 in 18, (preserving the notation). Consider any randomized scheme with two-parts memory. Denote by $C$ the cached memory (of size poly $(\log m)$ ) and by $B$ the main part of the memory of size $s$ (the scheme answers queries with one bit probe to $C$ ). Our aim is to prove a lower bound for $s$.

The proof is based on the bound for the size of cover-free families of sets proven by Dyachkov an Rykov [4. Let us remind that a family of sets $F$ is called called $r$-cover-free if $f_{0} \nsubseteq f_{1} \cup \ldots \cup f_{r}$ for all distinct $f_{0}, \ldots, f_{r} \in F$. 
First we take a large enough $\frac{1}{\varepsilon}$-cover free family $F$ of sets of size $n$ from the universe $\{1, \ldots, m\}$. The construction from [7, theorem 3.1] guarantees that there exists a family $F$ such that

$$
|F| \geq \frac{\left(\begin{array}{c}
m \\
\varepsilon n
\end{array}\right)}{\left(\begin{array}{c}
n \\
\varepsilon n
\end{array}\right)^{2}}=2^{\varepsilon n \log \frac{m}{n^{2}}+O(\log m)} .
$$

By assumption, each set $f \in F$ can be represented in our scheme by some pair $(B, C)$ (the main storage and the cached memory). Notice that

$$
|F|=2^{\varepsilon n \log \frac{m}{n^{2}}+O(\log m)} \gg 2^{|B|}=2^{\text {poly }(\log m)} .
$$

Hence, the exists some value $C$ and some $F^{\prime} \subset F$ of size

$$
\left|F^{\prime}\right| \geq|F| / 2^{\text {poly }(\log m)}=2^{\Omega(\varepsilon n \log m)} .
$$

such that all sets from the family $F^{\prime}$ share in our scheme the same value $C$ of the cached memory. Further, we repeat word for word the proof of theorem 2 from [18] with substitute $F^{\prime}$ instead of $F$.

\section{Conclusion.}

In this paper we constructed an effective probabilistic bit-probe scheme with one-sided error. The used space is close to the trivial information-theoretic lower bound $\Omega(n \log m)$. The scheme answers queries " $x \in A$ ?" with a small one-sided error and requires only poly-logarithmic (in the size of the universe) cached memory and one bit (two bits in the version with effective encoding) from the main part of the memory.

For reasonable values of parameters (for $n \gg$ poly $(\log m))$ the size of our scheme $O\left(n \log ^{2} m\right)$ with a cache of size poly $(\log m)$ is below the lower bound $\Omega\left(n^{2} \log m\right)$ proven in [18] for one-probe schemes with one-sided errors without cached data dependent on $A$. The gap between our upper bounds and the trivial lower bound is a $(\log m)$-factor.

The following questions remain open: How to construct a bit-probe memory scheme with one-sided error and effective encoding and decoding that requires to read only one bit from the main part of memory to answer queries? What is the minimal size of the cached memory required for a bit-probe scheme with one-sided error, with space of size $O(n \log m)$ ?

The author thanks Daniil Musatov for useful discussions, and anonymous referees of CSR2011 for deep and very helpful comments.

\section{References}

[1] B. Bloom. Space-time trade-offs in hash coding with allowable errors. Communications of ACM, 13(7) July 1970, 422-426. 
[2] M. S. Pinsker. On the complexity of a concentrator. In 7th International Teletrafc Conference, 1973, 318/1-318/4.

[3] L. A. Bassalygo and M. S. Pinsker. The complexity of an optimal nonblocking commutation scheme without reorganization. Problems of Information Transmission, 9, 1974, 64-66.

[4] A.G. Dyachkov and V.V. Rykov. Bounds on the length of disjunctive codes. Problems of information transmission, 18(3), 1982, 7-13.

[5] A.K. Chandra, L. Stockmeyer, U. Vishkin. Constant depth reducibility. SIAM Journal on Computing, 13, 1984, 423-429.

[6] M.L. Fredman, J. Komlós, E. Szemerédi. Storing a sparse table with $O(1)$ worst case access time. Journal of the Association for Computing Machinery, 31(3), 1984, 538-544.

[7] P. Erdös, P. Frankl, Z. Füredi. Families of nite sets in which no set is covered by the union of $\mathrm{r}$ others. Israel Journal of Mathematics, 51, 1985, 79-89.

[8] J. Håstad. Almost optimal lower bounds for small depth circuits. In Proc. of the 18th Annual ACM Symposium on Theory of Computing (STOC), $1986,6-20$.

[9] A. Siegel. On universal classes of fast high performance hash functions, their time-space trade-off, and their applications. Proc. of 30th IEEE Symposium on Foundations of Computer Science (FOCS), 1989, 20-25.

[10] N. Nisan. Pseudorandom generators for space-bounded computation. Combinatorica, 12(4), 1992, 449-461. Preliminary version: Proc. of the 22rd Annual ACM Symposium on Theory of Computing (STOC), 1990.

[11] A. Fiat, M. Naor, J.P. Schmidt, A. Siegel. Non-oblivious hashing, J. ACM 39, 1992, 764-782.

[12] N. Nisan, A. Wigderson, Hardness vs Randomness. J. Comput. Syst. Sci. 49(2), 1994, 149-167.

[13] N. Kahale. Eigenvalues and expansion of regular graphs. Journal of the ACM, 42(5), 1995, 1091-1106.

[14] A. Siegel. On universal classes of extremely random constant time hash functions and their time-space tradeoff. Technical Report TR1995-684, Courant Institute, New York University, April 1995.

[15] A. Brodnik, J.I. Munro. Membership in Constant Time and Minimum space. SIAM J. Comput. 28(5), 1999, 1627-1640.

[16] R. Pagh. Low Redundancy in Static Dictionaries with $O(1)$ Worst Case Lookup Time. In Proc. of the 26th International Colloquium on Automata, Languages and Programming (ICALP), 1999, 595-604. 
[17] J.S. Vitter. External memory algorithms and data structures. ACM Comput. Surv. 33(2), 2001, 209-271.

[18] H. Buhrman, P.B. Miltersen, J. Radhakrishnan, Venkatesh Srinivasan,Are bitvectors optimal? Siam J. on Computing 31(6), 2002, 1723-1744. Preliminary version: Proc. of the 32rd ACM Symposium on Theory of Computing (STOC), 2000, 449-458.

[19] A. Ta-Shma, C. Umans, D. Zuckerman. Loss-less Condensers, Unbalanced Expanders, and Extractors. In Proc. of the 33rd Annual ACM Symposium on Theory of Computing (STOC), 2001, 143-152.

[20] R. Pagh. On the Cell Probe Complexity of Membership and Perfect Hashing. In Proc. of the 33rd Annual ACM Symposium on Theory of Computing (STOC), 2001 425-432.

[21] M.R. Capalbo, O. Reingold, S.P. Vadhan, A. Wigderson. Randomness Conductors and Constant-Degree Lossless Expanders. In Proc. of the 34th Annual ACM Symposium on Theory of Computing (STOC), 2002, 659-668.

[22] D. Sivakumar. Algorithmic derandomization via complexity theory. In Proc. of the 34th Annual CM Symposium on Theory of Computing (STOC), 2002, 619-626.

[23] A. Östlin, R. Pagh. One-Probe Search. In Proc. of the 29th International Colloquium on Automata, Languages and Programming (ICALP), 2002, 439-450.

[24] A. Ta-Schma. Storing information with extractors. Information Processing Letters 83, 2002 267-274.

[25] L. Devroye, P. Morin. Cuckoo hashing: Further analysis. Information Processing Letters, 86, 2003, 215-219.

[26] R. Pagh, F. Rodler. Cuckoo Hashing. Journal of Algorithms, 51, 2004, 122144.

[27] S. Hoory, N. Linial, A. Wigderson. Expander graphs and their applications. Bulletin of the American Mathematical Society, 43(4) 2006, 439-561.

[28] J.S. Vitter. Algorithms and Data Structures for External Memory. Series on Foundations and Trends in Theoretical Computer Science, now Publishers, Hanover, MA, 2008.

[29] M. Braverman. Poly-logarithmic Independence Fools $\mathrm{AC}^{0}$ Circuits. In Proc. of the 27th IEEE Conference on Computational Complexity, 2009, 3-8.

[30] Y. Arbitman, M. Naor, G. Segev. De-amortized Cuckoo Hashing: Provable Worst-Case Performance and Experimental Results. In Proc. of the 36th International Colloquium on Automata, Languages and Programming (ICALP) 1, 2009, 107-118. 
[31] V. Guruswami, C. Umans, S. Vadhan. Unbalanced expanders and randomness extractors from Parvaresh-Vardy codes, Journal of the ACM, 56(4), 2009, 20:1-20:34.

[32] D. Musatov. Theorems about space-bounded Kolmogorov complexity obtained by "naive" derandomization. In Proc. 6th International Computer Science Symposium in Russia (CSR), 2011, 64-76. Prelim. version: arXiv: 1009.5108 (2010).

[33] M. David, P.A. Papakonstantinou, A. Sidiropoulos. How strong is Nisan's pseudorandom generator? 2010. Information Processing Letters, to appear. Electronic preprint (accessed September 14, 2011):

http://www.cs.toronto.edu/ papakons/pdfs/nisan_RNC.pdf 